Pacific Journal of Mathematic 


\section{INTEGRAL COMPARISON THEOREMS FOR THIRD ORDER LINEAR DIFFERENTIAL EQUATIONS}

\section{ERBE}

By means of a change of variable in the Riccati equation corresponding to the third order linear equation $L y \equiv$ $y^{\prime \prime \prime}+p(t) y^{\prime}+q(t) y=0$ a nonlinear integral equation is obtained which has a solution obtainable by successive approximations under certain conditions on $p$ and $q$. This technique allows one to obtain new sharp comparison theorems for $L y=0$. Several examples are given to illustrate the results.

Introduction. The third linear differential equation

$$
L y \equiv y^{\prime \prime \prime}+p(t) y^{\prime}+q(t) y=0
$$

where $p, q \in C[a, b), 0<a<b \leqq+\infty$, has a very extensive literature relating to the oscillatory and asymptotic behavior of its solutions, with much of the recent impetus coming from the work of Hanan [7], Lazer [10], Azbelev and Caljuk [1], and Barrett [2]. (See also Swanson [12].) In this paper a technique for obtaining some new sharp comparison theorems for (1.1) and a related equation

$$
L_{1} y \equiv y^{\prime \prime \prime}+p_{1}(t) y^{\prime}+q_{1}(t) y=0, \quad p_{1}, q_{1} \in C[a, b)
$$

will be introduced. Section 2 below will be devoted to some theorems which are consequences of more general results to be proved in $\S 3$. A comparison of the results obtained and their sharpness will also be discussed and illustrated by several examples.

2. Recall that equation (1.1) is said to be disconjugate on an interval $I$ in case no nontrivial solution has more than two zeros on $I$, counting multiplicity. If $I=[a,+\infty)$ (or $(a,+\infty)$ ), then $(1.1)$ is said to be oscillatory if it has at least one nontrivial oscillatory solution (i.e., a solution with an infinite number of zeros) and nonoscillatory iff all of its solutions are nonoscillatory (i.e., have finitely many zeros).

A useful comparison equation for third order linear equations is the Euler equation

$$
y^{\prime \prime \prime}+\alpha t^{-2} y^{\prime}+\beta t^{-3} y=0, \quad \alpha, \beta \text { real constants . }
$$

It is known (cf. [12]) that (2.1) is disconjugate on $(0,+\infty)$ iff $\alpha \leqq 1$ and $|\alpha+\beta| \leqq 2((1-\alpha) / 3)^{3 / 2}$. There are various tests for oscillation and disconjugacy using the Euler equation in conjunction with known 
comparison theorems. For example, Hanan [7] has shown that if the inequalities

$$
p(t) \geqq p_{1}(t), \quad q(t) \geqq q_{1}(t),
$$

and

$$
2 q(t)>p^{\prime}(t), \quad 2 q_{1}(t)>p_{1}^{\prime}(t)
$$

hold for all large $t$ and if $L y=0$ is nonoscillatory, then so is $L_{1} y=0$. Using (2.1) as a comparison equation, it was further shown in [7] that $L_{1} y=0$ is nonoscillatory if $p_{1} \in C^{\prime}[a,+\infty)$ and

(2.4) $p_{1}(t) \geqq 0,2 q_{1}(t)>p_{1}^{\prime}(t), t \in[\alpha,+\infty)$, and if there exists a number $k, 0<k<1$, with

$$
\begin{gathered}
\limsup _{t \rightarrow \infty} t^{2} p_{1}(t)<k \text { and } \\
\limsup _{t \rightarrow \infty} t^{3} q_{1}(t)<2((1-k) / 3)^{3 / 2}-k .
\end{gathered}
$$

In particular, if $p_{1} \equiv 0$ and $q_{1}$ is of constant sign, then $L_{1} y=0$ is oscillatory if

$$
\liminf _{t \rightarrow \infty} t^{3}\left|q_{1}(t)\right|>\frac{2}{3 \sqrt{3}}
$$

and is nonoscillatory if

$$
\limsup _{\rightarrow \infty} t^{3}\left|q_{1}(t)\right|<\frac{2}{3 \sqrt{3}} .
$$

It will be seen that examples of equations can be given with $\lim \sup _{t \rightarrow \infty} q_{1}(t)=+\infty$ which are, nevertheless, disconjugate on $[a,+\infty)$. This is a consequence of the fact that the criteria obtained here are of an integral type. Theorems of this type seem to be scarce in the literature although some results in this direction were obtained by the author in [4] for the case $p \equiv p_{1} \equiv 0$. To motivate the more general results of $\S 3$ (which involve more complicated hypotheses) several special cases will now be stated and briefly discussed. More elaborate examples will be given in $\S 3$, along with the proofs (see Remark 3.8). To simplify the statements of the theorems, the following notation will be used:

$$
\begin{aligned}
\hat{P}_{1}(t) & \equiv \int_{a}^{t}(t-s)(s-a)^{2} p_{1}(s) d s, \\
\hat{P}(t) & \equiv \int_{a}^{t}(t-s)(s-a)^{2} p(s) d s, \quad t \geqq a
\end{aligned}
$$




$$
\begin{aligned}
\hat{Q}_{1}(t) & \equiv \int_{a}^{t}(t-s)(s-a)^{3} q_{1}(s) d s, \\
\hat{Q}(t) & \equiv \int_{a}^{t}(t-s)(s-a)^{3} q(s) d s, \quad t \geqq a
\end{aligned}
$$

THEOREM 2.1. Let $p \equiv p_{1} \equiv 0, q \geqq 0$ and $\not \equiv 0$ on any subinterval, $t \geqq a$, and assume $L y=y^{\prime \prime \prime}+q y=0$ is disconjugate on $[a,+\infty)$. Assume further that

$$
\begin{aligned}
& \int_{a}^{t}(t-s)(s-a)^{3} q_{1}^{+}(s) d s \leqq \hat{Q}(t), \quad t>a \quad \text { and } \\
& \int_{a}^{t}(t-s)(s-a)^{3} q_{1}^{-}(s) d s \leqq \hat{Q}(t), \quad t>a,
\end{aligned}
$$

where $q_{1}^{+}(t)=\max \left[q_{1}(t), 0\right], q_{1}^{-}(t)=\max \left[-q_{1}(t), 0\right]$. Then $L_{1} y=0$ is disconjugate on $[a,+\infty)$.

THEOREM 2.2. $L_{1} y=0$ is disconjugate on $[a,+\infty)$ in case

$$
-(a / \sqrt{3})(t-a) \leqq P_{1}(t)+Q_{1}(t) \leqq(t-a)^{2} /(3 \sqrt{3}), \quad t>a
$$

and

(2.14) $0 \leqq t^{2} p_{1}(t) \leqq k v_{0}(t), t>a$ for some $0<k<\frac{1}{2}+\frac{1}{\sqrt{3}}$, where

$$
\begin{gathered}
t^{2} v_{0}(t)=a^{2}\left(1-\frac{1}{\sqrt{3}}\right)+\frac{a}{\sqrt{3}}(t-a)+P_{1}(t)+Q_{1}(t), \quad \text { and } \\
P_{1}(t)=\int_{a}^{t}(t-s) s^{2} p_{1}(s) d s, \quad Q_{1}(t)=\int_{a}^{t}(t-s) s^{3} q_{1}(s) d s .
\end{gathered}
$$

The above results are sharp in some sense because of the Euler equation. In fact, Theorem 2.2 is obtained from Theorem 3.3 by using the nonoscillatory Euler equation (2.1) with $\alpha=0$ and $\beta=$ $2 / 3 \sqrt{3}$ as a comparison equation. As special cases of the above theorems (and the more general results in §3) are included a few of the results of Hanan [7] mentioned earlier. In particular, the following result generalizes (2.8):

Corollary 2.3. If $q(t)$ has constant sign, then $y^{\prime \prime \prime}+q(t) y=0$ is disconjugate on $[a,+\infty)$ if

$$
\left|\int_{a}^{t}(t-s) s^{3} q(s) d s\right| \leqq(t-a)^{2} / 3 \sqrt{3}, \quad t>a .
$$

3. Proofs, generalizations, and examples. In this section, the 
more general results needed to obtain the theorems in $\S 2$ will be stated and proved. Examples illustrating the results will also be given.

The Riccati equation corresponding to $L y=0$ is

$$
r^{\prime \prime}+3 r r^{\prime}+r^{3}+p(t) r+q(t)=0, \quad r=\frac{y^{\prime}}{y} .
$$

It is known (cf. [5]) that $L y=0$ is disconjugate on an interval $I$ iff there exist $\alpha, \beta \in C^{2}(I)$ (lower and upper solutions, respectively) with $\alpha(t)<\beta(t)$ on $I$ and such that

$$
\alpha^{\prime \prime}+f\left(t, \alpha, \alpha^{\prime}\right) \geqq 0 \geqq \beta^{\prime \prime}+f\left(t, \beta, \beta^{\prime}\right), \quad t \in I,
$$

where $f\left(t, r, r^{\prime}\right)=3 r r^{\prime}+r^{3}+p(t) r+q(t)$. Using this, many effective criteria may be obtained for disconjugacy of $L y=0$ based, for example, on the separation of the roots of the equation $\sigma(\rho, t)=$ $\rho^{3}+p(t) \rho+q(t)=0$ by real constants (cf. [3], [8], and [9]).

The change of variable $w=2-t r$ transforms (3.1) into

$$
t^{2} w^{\prime \prime}+4 t w^{\prime}-3 t w w^{\prime}=3 w^{2}-2 w-w^{3}+t^{2} p(t)(2-w)+t^{3} q(t)
$$

There are several reasons for this particular change of variable. The Euler equation (2.1) (in the disconjugate case) always has a solution $t^{r}$ with $1<\gamma<2$, so that the corresponding $w$ satisfies $0<w<1$, in which range the kernel of the integral equation corresponding to (3.2) is monotone increasing in $w$. Moreover, this property is shared by many other disconjugate equations which may be used as comparison equations. The idea here is to solve the equation (3.2) by a monotone successive approximations technique applied to the corresponding integral equation. This leads to lower and upper solutions for (3.1) and hence disconjugacy. The integral equation corresponding to (3.2) (obtained by integration by parts twice) is

$$
\begin{aligned}
t^{2} w=g_{1}(a)+g_{2}(a)(t-a) & +\int_{a}^{t}(t-s) H(w, s) d s+P(t)+Q(t) \\
& +\frac{3}{2} \int_{a}^{t} s w^{2} d s
\end{aligned}
$$

where

$$
\begin{gathered}
H(w, s)=w^{2}\left(\frac{3}{2}-w\right)-s^{2} p(s) w, \\
g_{1}(a)=a^{2} w(a), g_{2}(a)=2 a w(a)+a^{2} w^{\prime}(a)-\frac{3}{2} a w^{2}(a), \quad \text { and }
\end{gathered}
$$




$$
P(t)=\int_{a}^{t}(t-s) s^{2} p(s) d s, Q(t)=\int_{a}^{t}(t-s) s^{3} q(s) d s
$$

Thus, $w=w(t)$ is a solution of (3.2) iff $w$ solves (3.3). It will now be shown that, under suitable conditions, a solution of (3.3) can be obtained by successive approximations. Given the solution $w=w(t)$, let the sequence $w_{n}=w_{n}(t), n=0,1,2, \cdots$ be defined by

$$
\left\{\begin{array}{l}
t^{2} w_{0}(t)=g_{1}(a)+g_{2}(a)(t-a)+P(t)+Q(t) \\
t^{2} w_{n}(t)=t^{2} w_{0}(t)+\int_{a}^{t}(t-s) H\left(w_{n-1}, s\right) d s+\frac{3}{2} \int_{a}^{t} s w_{n-1}^{2} d s, \quad n \geqq 1 .
\end{array}\right.
$$

LEMMA 3.1. Let $w=w(t)$ be a solution of (3.3) with $0<w(t)<1$ on $[a, b), 0<a<b \leqq+\infty$, and assume

$$
g_{2}(a)(t-a)+P(t)+Q(t) \geqq 0, \quad t>a .
$$

Assume further that

$$
H\left(w_{0}(t), t\right) \geqq 0, \quad t \geqq a \quad \text { and }
$$

(3.10) $H_{u}(u, t) \geqq 0$ for each fixed $t$ and $w_{0}(t) \leqq u \leqq w(t)$,

where $H_{u}(u, t) \equiv \partial H / \partial u(u, t)=3 u(1-u)-t^{2} p(t)$.

Then the sequence $\left\{w_{n}\right\}_{n=0}^{\infty}$ converges uniformly to $w$ on each compact subset of $[a, b)$.

Proof. By (3.8) it is seen that $t^{2} w_{0}(t)>0, t \geqq a$. Also,

$$
t^{2}\left(w_{1}(t)-w_{0}(t)\right)=\int_{a}^{t}(t-s) H\left(w_{0}, s\right) d s+\frac{3}{2} \int_{a}^{t} s w_{0}^{2} d s>0 .
$$

Hence, for $n>1$

$$
\begin{aligned}
t^{2}\left(w_{n}(t)-w_{n-1}(t)\right)= & \int_{a}^{t}(t-s)\left(H\left(w_{n-1}, s\right)-H\left(w_{n-2}, s\right)\right) d s \\
& +\frac{3}{2} \int_{a}^{t} s\left(w_{n-1}^{2}-w_{n-2}^{2}\right) d s>0
\end{aligned}
$$

for $t>a$, by induction and (3.10), provided $w_{n}(t)<w(t), t>a$. But

$$
\begin{gathered}
t^{2}\left(w(t)-w_{0}(t)\right)=\int_{a}^{t}(t-s) H(w, s) d s+\frac{3}{2} \int_{a}^{t} s w^{2} d s>0 \text { and } \\
t^{2}\left(w(t)-w_{n}(t)\right)=\int_{a}^{t}(t-s)\left[H(w, s)-H\left(w_{n-1}, s\right)\right] d s \\
+\frac{3}{2} \int_{a}^{t} s\left(w^{2}-w_{n-1}^{2}\right) d s>0
\end{gathered}
$$


again by induction, using (3.9), (3.10). It follows therefore, by the Monotone Convergence Theorem, that the sequence $\left\{w_{n}\right\}_{n=0}^{\infty}$ converges,

$$
\lim _{n \rightarrow \infty} w_{n}(t) \equiv w^{*}(t) \leqq w(t), \quad \text { for each } t,
$$

and $w^{*}(t)$ satisfies equations (3.3) with the same initial conditions as $w(t)$. Hence, $w^{*}(t) \equiv w(t)$. By Dini's Theorem, the convergence is uniform on compact subset of $[a, b)$. This completes the proof.

REMARK 3.2. If $p(t) \leqq 0$, then (3.9), (3.10) hold trivially. It will be seen that, for purposes of applying Lemma 3.1, w(t) and $w_{0}(t)$ are often explicitly computable and that as a consequence, the validity of (3.9) and (3.10) becomes a simple matter to verify. Note also that if $q(t) \geqq 0, t \geqq a$, then the existence of a solution of $w$ of (3.3), with $0<w(t)<1$ implies that (1.1) is disconjugate. (Here $\alpha \equiv 0$ is a lower solution of (3.1) and $r(t) \equiv(2-w) / t>0$ is an upper solution.)

It will now be shown that, under suitable hypotheses, the existence of a solution of (3.3) on $[a, b)$ can be used to obtain the existence of a solution of the integral equation corresponding to $L_{1} y=0$. The Riccati equation corresponding to $L_{1} y=0$ is

$$
\rho^{\prime \prime}+3 \rho \rho^{\prime}+\rho^{3}+p_{1}(t) \rho+q_{1}(t)=0, \quad \rho=\frac{y^{\prime}}{y}
$$

and the integral equation obtained via the transformation $v=2-t \rho$ is

$$
\begin{aligned}
t^{2} v= & G_{1}(a)+G_{2}(a)(t-a)+\int_{a}^{t}(t-s) H_{1}(v, s) d s+P_{1}(t) \\
& +Q_{1}(t)+\frac{3}{2} \int_{a}^{t} s v^{2} d s
\end{aligned}
$$

where

$$
\begin{gathered}
H_{1}(v, s)=v^{2}\left(\frac{3}{2}-v\right)-s^{2} p_{1}(s) v, \\
P_{1}(t)=\int_{a}^{t}(t-s) s^{2} p_{1}(s) d s, Q_{1}(t)=\int_{a}^{t}(t-s) s^{3} q_{1}(s) d s, \quad \text { and } \\
G_{1}(a)=a^{2} v(a), G_{2}(a)=a v(a)\left(2-\frac{3}{2} v(a)\right)+a^{2} v^{\prime}(a) .
\end{gathered}
$$

Thus, if $v=v(t)$ solves (3.12) on $[a, b)$ then $\rho=\rho(t)$ defined by $\rho(t)=(2-v(t)) / t$ is a solution of (3.11) and therefore $y=\exp \int_{a}^{t} \rho(s) d s$ is a positive solution of $L_{1} y=0$. 
Given a solution $w=w(t)$ of (3.3) with $0<w(t)<1$ on $[a, b)$, $0<a<b \leqq+\infty$, let the sequence $\left\{v_{n}(t)\right\}_{n=0}^{\infty}$ be defined for $a \leqq t<b$ by

$$
\left\{\begin{array}{l}
t^{2} v_{0}(t)=g_{1}(a)+g_{2}(a)(t-a)+P_{1}(t)+Q_{1}(t), \\
t^{2} v_{n}(t)=t^{2} v_{0}(t)+\int_{a}^{t}(t-s) H_{1}\left(v_{n-1}, s\right) d s+\frac{3}{2} \int_{a}^{t} s v_{n-1}^{2} d s, n \geqq 1,
\end{array}\right.
$$

where $g_{1}(a), g_{2}(a)$ are as in (3.5).

THEOREM 3.3. Let $w=w(t)$ be a solution of (3.3) with $0<w(t)<$ 1 on $[a, b), 0<a<b \leqq+\infty$, and assume

$$
g_{2}(a)(t-a)+P_{1}(t)+Q_{1}(t) \geqq 0, \quad t>a
$$

where $g_{2}(a)$ is as in (3.5). Assume further that

$$
\begin{gathered}
p(t) \leqq p_{1}(t) \quad \text { and } \\
P_{1}(t)+Q_{1}(t) \leqq P(t)+Q(t), \quad t \geqq a .
\end{gathered}
$$

Assume also that

$$
H_{1}\left(v_{0}(t), t\right) \geqq 0, \quad t \geqq a \quad \text { and }
$$

$$
\frac{\partial H_{1}}{\partial v}(v, t) \geqq 0 \text { for each fixed } t \text { and } v_{0}(t) \leqq v \leqq w(t) \text {. }
$$

Then the sequence $\left\{v_{n}\right\}_{n=0}^{\infty}$ defined in (3.16) converges, uniformly on compact subsets of $[a, b)$, to a solution $v=v(t)$ of (3.12) with $0<$ $v(t) \leqq w(t)$ on $[a, b)$.

Proof. Notice first that (3.20) and (3.21) imply that $H(w, t)$ satisfies (3.9) and (3.10), provided $v_{0}(t) \leqq w_{0}(t)$.

As in Lemma $3.1, t^{2} v_{0}(t)>0, t \geqq a$ by $(3.17)$ and $v_{n}(t)>v_{n-1}(t)$, $n=1,2, \cdots$ by induction. Furthermore, if the $w_{n}$ are as defined in (3.7), then $t^{2}\left(w_{0}(t)-v_{0}(t)\right) \geqq 0$ by (3.19) and for $n \geqq 1$ we get after some rearranging

$$
\begin{aligned}
t^{2}\left(w_{n}(t)-v_{n}(t)\right)= & \int_{a}^{t}(t-s)\left(H_{1}\left(w_{n-1}, s\right)-H_{1}\left(v_{n-1}, s\right)\right) d s \\
& +\int_{a}^{t}(t-s) s^{2} w_{n-1}\left(p_{1}(s)-p(s)\right) d s+P(t)+Q(t) \\
& -P_{1}(t)-Q_{1}(t)+\frac{3}{2} \int_{a}^{t} s\left(w_{n-1}^{2}-v_{n-1}^{2}\right) d s \geqq 0
\end{aligned}
$$

by (3.21), (3.19), (3.18) and induction. Hence, $\lim _{n \rightarrow \infty} v_{n}(t) \equiv v(t) \leqq w(t)$, as in Lemma 3.1, the convergence being uniform on compact subsets of $[a, b)$, and $v(t)$ is a solution of (3.12). This completes the proof. 
REMARK 3.4. Notice that the solution $v=v(t)$ of (3.12) obtained in the previous theorem satisfies $v(a)=g_{1}(a) / a^{2}=w(a)$. If, in the definition of the sequence $\left\{v_{n}\right\}$ in (3.16), $g_{1}(a)$ is replaced by $g(a)$, $0<g(a)<g_{1}(a)$ (i.e., $v_{0}(t)$ is replaced by $v_{0}^{*}(t)$, where $v_{0}^{*}(t)$ is defined as in (3.16) with $g(a)$ instead of $g_{1}(a)$ ) then Theorem 2.3 may be applied to the new sequence $\left\{v_{n}^{*}\right\}$ so obtained, provided that (3.20) and (3.21) hold with $v_{0}$ replaced by $v_{0}^{*}$. Therefore, a second solution $v^{*}(t)$ may be obtained with $0<v^{*}(t) \leqq v(t)$ on $[a, b)$. Moreover, since $v^{*}(a)<v(a)$, it follows (by uniqueness of solutions of initial value problems) that $v^{*}(t)<v(t)$ on $[a, b)$. Thus, $\alpha(t) \equiv(2-v(t)) / t<$ $\beta(t) \equiv\left(2-v^{*}(t)\right) / t$ are lower and upper solutions of (3.11) so that $L_{1} y=0$ is disconjugate on $[a, b)$. This may be summarized as

CoROllary 3.5. $L_{1} y=0$ is disconjugate on $[a, b)$ provided that the hypotheses of Theorem 3.3 hold and, in addition, that (3.20) and (3.21) hold with $v_{0}(t)$ replaced by $v_{0}^{*}(t)$, where $v_{0}^{*}(t)$ is defined as in (3.16) with $g_{1}(a)$ replaced by $g(a)$, for some $0<g(a)<g_{1}(a)$.

Before stating the next theorem the following definition and some notation will be introduced.

Definition 3.6. $L y=y^{\prime \prime \prime}+p y^{\prime}+q y=0$ is said to have property $A$ on the interval $[a, b)$ in case the solution with $y(a)=y^{\prime}(a)=$ $y^{\prime \prime}(a)-1=0$ satisfies $y>0, y^{\prime}>0, y^{\prime \prime}>0, y^{\prime \prime \prime} \leqq 0$ on $(a, b)$, and $y^{\prime \prime \prime} \not \equiv 0$ on any subinterval of $(a, b)$.

A sufficient condition for property $A$ to hold on $[a,+\infty$ ) is (cf. [10], Lemma 3.1) that $L y=0$ be disconjugate on $[a,+\infty)$ and $p \geqq 0$, $q \geqq 0,2 q-p^{\prime} \geqq 0$ and $\neq \equiv 0$ on any subinterval of $[a,+\infty)$. Another sufficient condition is (cf. [6]) that $L y=0$ be disconjugate on $[a,+\infty$ ) and $p \geqq 0, q>0$, and $p / q$ nondecreasing on $[a,+\infty)$.

If $L y=0$ has property $A$ and $y>0, y^{\prime}>0, y^{\prime \prime}>0, y^{\prime \prime \prime} \leqq 0$ on $(a, b)$, then $r(t)=y^{\prime}(t) / y(t), t>a$ satisfies $\lim _{t \rightarrow a+}(t-a) r(t)=2$. Therefore, if $\hat{w}=\widehat{w}(t)$ is defined by

$$
\begin{aligned}
& \hat{w}(t)=2-(t-a) r(t), t>a \\
& w(a)=0
\end{aligned}
$$

then noting that the function $F(t) \equiv(t-a) y^{\prime}(t)-y(t)$ satisfies $F(a)=$ $0, F^{\prime}>0, t>a$ it follows that $1<(t-a) y^{\prime}(t) / y(t), t>a$, i.e., that $\hat{w}(t)<1, t>a$. Similarly, by considering the function $F_{1}(t)=$ $(t-a) y^{\prime}(t)-2 y(t)$ which satisfies $F_{1}(a)=F_{1}^{\prime}(a)=0$ and $F_{1}^{\prime \prime} \leqq 0$ and $\not \equiv 0$ on any subinterval $t \geqq a$, it follows that, $F_{1}<0, t \geqq a$, which implies $0<\hat{w}(t), t>a$. It follows that $\hat{w} \in C[a, b) \cap C^{2}(a, b)$. Furthermore,

$$
\lim _{t \rightarrow a+}(t-a) \hat{w}^{\prime}(t)=\lim _{t \rightarrow a+}(t-a)^{2} \widehat{w}^{\prime \prime}(t)=0
$$


by l'Hospital's rule and some elementary manipulations. Therefore $\hat{w}$ satisfies the equation

$$
\left\{\begin{array}{l}
(t-a)^{2} \hat{w}^{\prime \prime}+4(t-a) \hat{w}^{\prime}-3(t-a) \hat{w} \hat{w}^{\prime} \\
=-\hat{w}^{3}+3 \hat{w}^{2}-2 \hat{w}+(t-a)^{2} p(t)(2-\hat{w})+(t-a)^{3} q(t), \quad t>a .
\end{array}\right.
$$

By (3.23) and (3.24) the corresponding integral equation is

$$
\begin{aligned}
(t-a)^{2} \hat{w}(t)= & \int_{a}^{t}(t-s) \hat{H}(\hat{w}, s) d s+\hat{P}(t)+\hat{Q}(t) \\
& +\frac{3}{2} \int_{a}^{t}(s-a) \hat{w}^{2} d s
\end{aligned}
$$

where

$$
\hat{H}(\hat{w}, t)=\widehat{w}^{2}\left(\frac{3}{2}-\widehat{w}\right)-(t-a)^{2} p(t) \hat{w}, \hat{P}(t)=\int_{a}^{t}(t-s)(s-a)^{2} p(s) d s
$$

and $\hat{Q}(t)=\int_{a}^{t}(t-s)(s-a)^{3} q(s) d s$.

Similar notation $\hat{P}_{1}(t), \hat{Q}_{1}(t), \hat{H}_{1}(\hat{v}, t)$ will be used in the integral equation corresponding to $L_{1} y=0$ :

$$
\begin{aligned}
(t-a)^{2} \hat{v}(t)= & \int_{a}^{t}(t-s) \hat{H}_{1}(\hat{v}, s) d s+\hat{P}_{1}(t)+\hat{Q}_{1}(t) \\
& +\frac{3}{2} \int_{a}^{t}(s-a) \hat{v}^{2} d s .
\end{aligned}
$$

The sequences $\left\{\hat{v}_{n}\right\}_{n=0}^{\infty},\left\{\hat{w}_{n}\right\}_{n=0}^{\infty}$ are defined for $t>a$ by

$$
\left\{\begin{aligned}
(t-a)^{2} \hat{w}_{0}(t)= & \hat{P}(t)+\hat{Q}(t), \\
(t-a)^{2} \hat{w}_{n}(t)= & \int_{a}^{t}(t-s) \hat{H}\left(\hat{w}_{n-1}, s\right) d s \\
& +\frac{3}{2} \int_{a}^{t}(t-s) \hat{w}_{n-1}^{2} d s+\hat{P}(t)+\hat{Q}(t)
\end{aligned}\right.
$$

and

$$
\begin{aligned}
(t-a)^{2} \hat{v}_{0}(t)= & \hat{P}_{1}(t)+\hat{Q}_{1}(t) \\
(t-a)^{2} \hat{v}_{n}(t)= & \int_{a}^{t}(t-s) \hat{H}_{1}\left(\hat{v}_{n-1}, s\right) d s \\
& +\frac{3}{2} \int_{a}^{t}(t-s) \hat{v}_{n-1}^{2} d s+\hat{P}_{1}(t)+\hat{Q}_{1}(t) .
\end{aligned}
$$

The following theorem may now be proved:

THEOREM 3.7. Assume $L y=0$ has property A on $[a, b)$ and that 


$$
0 \leqq \hat{P}_{1}(t)+\hat{Q}_{1}(t) \leqq \hat{P}(t)+\hat{Q}(t), \quad t \geqq a .
$$

Assume further that $p(t) \leqq p_{1}(t)$ and that

$$
\begin{aligned}
& \hat{H}_{1}\left(\widehat{v}_{0}(t), t\right)=\hat{v}_{0}^{2}\left(\frac{3}{2}-\widehat{v}_{0}\right)-(t-a)^{2} p_{1}(t) \hat{v}_{0} \geqq 0 \text { and } \\
& \frac{\partial \hat{H}_{1}}{\partial v}(\hat{v}, t)=3 \hat{v}(1-\hat{v})-(t-a)^{2} p_{1}(t) \geqq 0 \text { for each fixed } t \text { and } \\
& \widehat{v}_{0}(t) \leqq \hat{v} \leqq \hat{w} .
\end{aligned}
$$

Then equation (3.26) has a solution $\hat{v}=\hat{v}(t)$ with $0<\hat{v}(t) \leqq \hat{w}(t)$ on $(a, b)$ and $L_{1} y=0$ has a positive solution on $(a, b)$. Further, if $q_{1} \geqq 0, t \geqq a$, then $L_{1} y=0$ is disconjugate on $[a, b)$.

Proof. The proof is similar to the proof of Theorem 3.3. The sequence $\left\{\hat{w}_{n}(t)\right\}_{n=0}^{\infty}$ converges monotonically to $\hat{w}(t)$ (uniformly on compact subsets of $[a, b))$. Likewise the sequence $\left\{v_{n}(t)\right\}_{n=0}^{\infty}$ is monotone increasing for each $t$ and satisfies

$$
\widehat{w}_{n}(t)-\hat{v}_{n}(t) \geqq 0, \quad n=0,1,2, \cdots .
$$

Therefore, $\left\{\hat{v}_{n}(t)\right\}_{n=0}^{\infty}$ converges to a solution $\hat{v}(t)$ of (3.26) with $0<\hat{v}(t) \leqq \hat{w}(t)<1, t>a$. Hence, $y_{1}(t) \equiv \exp \left(\int_{t_{0}}^{t} \rho(s) d s\right)$, where $\rho(t)=$ $(2-\hat{v}(t)) /(t-a)$, and $a<t_{0}<b$, is a positive solution of $L_{1} y=0$ on $(a, b)$. Finally if $q_{1} \geqq 0, t \geqq a$, then $\alpha(t) \equiv 0$ and $\beta(t) \equiv \rho(t)$ are lower and upper solutions of (3.11) so that $L_{1} y=0$ is disconjugate on $(a, b)$ and hence on $[a, b)$.

REMARK 3.8. The proof of the theorems in $\S 2$ follow from the previous results:

Proof of Theorem 2.1. By the comments following Definition 3.6 , it follows that $L y=0$ has property A. Therefore, conditions (2.11) and (2.12) imply that both $L_{1}^{+} y \equiv y^{\prime \prime \prime}+q_{1}^{+} y=0$ and $L_{1}^{-} y \equiv$ $y^{\prime \prime \prime}+q_{1}^{-} y=0$ are disconjugate on $[a,+\infty)$, by Theorem 3.7. Hence, the equation $y^{\prime \prime \prime}-q_{1}^{-} y=0$ is also disconjugate on $[a,+\infty$ ) (cf. [7], Theorem 4.7). Since $-q_{1}^{-}(t) \leqq q_{1}(t) \leqq q_{1}^{+}(t)$, it follows (cf. [11]) that $L_{1} y=0$ is disconjugate on $[a,+\infty)$.

Proof of Theorem 2.2. Let $\alpha=0, \beta=2 / 3 \sqrt{3}$ in the Euler equation (2.1), (i.e., $p(t) \equiv 0, q(t)=(2 / 3 \sqrt{3}) t^{-3}$ in Theorem 3.3. Then (2.1) has the solution $t^{r}, \gamma=1+1 / \sqrt{3}$, and the corresponding solution of $(3.3)$ is $w \equiv 2-\gamma=1-1 / \sqrt{3}<1$. Also, $g_{1}(a)=a^{2} \gamma_{0}$ and $g_{2}(a)=$ $a / \sqrt{3}$ so that if (2.13) holds, then (3.17) and (3.19) in Theorem 3.3 
hold. Now (2.14) implies, on the one hand, that (3.18) holds and on the other, since $t^{2} p_{1}(t) \leqq k v_{0}(t), 0<k<1 / 2+1 / \sqrt{3}$, it follows that (3.20) and (3.21) also hold. To see this, notice that $\left(3 / 2-v_{0}(t)\right) \geqq$ $(3 / 2-w(t))=1 / 2+1 / \sqrt{3}$ so that $t^{2} p_{1}(t) \leqq k v_{0}(t)<v_{0}(t)\left(3 / 2-v_{0}(t)\right)$, if $0<k<1 / 2+1 / \sqrt{3}$. Similarly, (3.21) holds if $3 v(1-v) \geqq t^{2} p_{1}(t)$ for $v_{0}(t) \leqq v \leqq w(t)=1-1 / \sqrt{3}<1 / 2$. But since the function $f(v)=$ $3 v(1-v)$ is increasing for $0<v<1 / 2$ it suffices to have

$$
3 v_{0}(t)\left(1-v_{0}(t)\right) \geqq t^{2} p_{1}(t)
$$

in order to satisfy (3.21). Again, since $1-v_{0}(t) \geqq 1-w(t)=1 / \sqrt{3 \text {, }}$ it follows that $3 v(1-v) \geqq \sqrt{3} v_{0}(t)>k v_{0}(t) \geqq t^{2} p_{1}(t)$. Therefore, all of the conditions of Theorem 3.3 hold. Furthermore, if $0<g(a)<$ $g_{1}(a)$ is chosen so that $(1 / 2+1 \sqrt{3}) g(a)>k g_{1}(a)$, then (2.14) implies that (3.20) and (3.21) hold with $v_{0}(t)$ replaced by $v_{0}^{*}(t)$, where $v_{0}^{*}(t)$ is defined as in (3.16) with $g_{1}(a)$ replaced by $g(a)$. Hence, Corollary 3.5 implies that $L_{1} y=0$ is disconjugate on $[a, b)$. This completes the proof of Theorem 2.2.

Proof of Corollary 2.3. If $q(t)$ has constant sign, it may be assumed that $q \geqq 0$ since the equation $y^{\prime \prime \prime}+q(t) y=0$ is disconjugate iff $y^{\prime \prime \prime}-q(t) y=0$ is disconjugate. Suppose then that (2.17) holds, $q \geqq 0$. The conclusion then follows immediately from Theorem 2.2.

Several additional examples will now be considered in order to more fully illustrate the results.

EXAMPLe 3.9. Consider the Euler equation (2.1) with $-2<\alpha \leqq 1$ and $\beta=2((1-\alpha) / 3)^{3 / 2}-\alpha$, so that $(2.1)$ is disconjugate and has the solution $t^{\gamma}, \gamma=1+((1-\alpha) / 3)^{1 / 2}$. Then $w(t) \equiv 2-\gamma=1-\left((1-\alpha)_{/} / 3\right)^{1 / 2}$ satisfies $0<w<1, g_{1}(a)=a^{2} \gamma, g_{2}(a)=a\left(\alpha / 2+((1-\alpha) / 3)^{1 / 2}\right)$. Therefore, (3.17), (3.18), and (3.19) of Theorem 3.3 hold if

$$
\begin{aligned}
\alpha \leqq t^{2} p_{1}(t), t & \geqq a \text { and } \\
-a\left(\frac{\alpha}{2}+\left(\frac{1-\alpha}{3}\right)^{1 / 2}\right)(t-a) & \leqq P_{1}(t)+Q_{1}(t) \\
& \leqq\left(\frac{1-\alpha}{3}\right)^{3 / 2}(t-a)^{2}, \quad t \geqq a .
\end{aligned}
$$

Further, since $v_{0}(t)\left(3 / 2-v_{0}(t)\right) \geqq v_{0}(t)(3 / 2-w(t))=\left(1 / 2+((1-\alpha) / 3)^{1 / 2}\right) v_{0}(t)$ and $3 v(1-v) \geqq 3 v_{0}(t)(1-w(t))=\sqrt{3(1-\alpha)} v_{0}(t)$, for $v_{0}(t) \leqq v$, it follows that both (3.20) and (3.21) hold if

$$
t^{2} p_{1}(t) \leqq k v_{0}(t), \quad \text { where } k \text { satisfies }
$$

$$
k<k_{0} \equiv \min \left[\sqrt{3(1-\alpha)}, \frac{1}{2}+\left(\frac{1-\alpha}{3}\right)^{1 / 2}\right] \text {. }
$$


Finally, if $0<g(a)<g_{1}(a)$ is chosen so that

$$
k_{0} g(a)>k g_{1}(a)
$$

then (3.34) implies that (3.20) and (3.21) hold with $v_{0}(t)$ replaced by $v_{0}^{*}(t)$ where $v_{0}^{*}(t)$ is defined as in (3.16) with $g(a)$ replacing $g_{1}(a)$. It is worth noting that if $\alpha>0$ and if (3.32) and (3.34) hold, then

$$
\alpha t^{2} \leqq t^{4} p_{1}(t) \leqq k t^{2} v_{0}(t) \leqq k\left(\frac{1-\alpha}{3}\right)^{3 / 2} t^{2}+0(t), \quad t \rightarrow \infty
$$

Thus, it is necessary that

$$
\alpha<k\left(\frac{1-\alpha}{3}\right)^{3 / 2}
$$

so that by (3.35) it is necessary that

$$
\alpha<\frac{(1-\alpha)^{2}}{3} \text { and } \alpha<\frac{(1-\alpha)^{2}}{9}+\frac{1}{2}\left(\frac{1-\alpha}{3}\right)^{3 / 2} \text {. }
$$

To summarize this example: $L_{1} y=0$ is disconjugate on $[a,+\infty)$ if there exists $\alpha,-2<\alpha<1$ so that (3.32)-(3.36) and (3.39) hold.

ExAMPLE 3.10. Consider the Euler equation (2.1) again and assume $-2<\alpha<0$ and

$$
\alpha \leqq t^{2} p_{1}(t) \leqq 0, \quad t \geqq a .
$$

In this case conditions (3.20) and (3.21) hold trivially so that $L_{1} y=0$ is disconjugate on $[a,+\infty)$ in case (3.40) and (3.33) hold. As a specific example, let $\alpha=-1 \equiv t^{2} p_{1}(t)$ and let

$$
t^{3} q_{1}(t) \equiv k_{1}+1-\left(k_{1}+k_{2}\right) \cos (t-a),
$$

where $0<a<+\infty, k_{1}=2(2 / 3)^{3 / 2}, k_{2}=a\left((2 / 3)^{1 / 2}-1 / 2\right)$. Then $t^{2} p_{1}(t)+$ $t^{3} q_{1}(t)=k_{1}-\left(k_{1}+k_{2}\right) \cos (t-a)$ so that $P_{1}(t)+Q_{1}(t)=\left(k_{1} / 2\right)(t-a)^{2}+$ $\left(k_{1}+k_{2}\right)(\cos (t-a)-1) \leqq(2 / 3)^{3 / 2}(t-a)^{2}, t>a$. Also, $P_{1}(t)+Q_{1}(t)+$ $k_{2}(t-a)>0, t>a$, and hence $L_{1} y=0$ is disconjugate on $[a,+\infty)$. This behavior may not be deduced from any other criteria known to the author.

The examples given above are, of course, sharp by virtue of the Euler equation and, as noted, reasonably simple examples may be found whose disconjugate behavior may not be determined by known criteria.

EXAMPLE 3.11. To find an example of a disconjugate equation $L_{1} y=0$ with $\lim \sup _{t \rightarrow \infty} q_{1}(t)=+\infty$ is straightforward. For convenience assume $p_{1} \equiv 0$ and let $q_{1} \in C[1,+\infty)$ with $q_{1}(t)>0$ and 


$$
q_{1}(n)=n \text { and } \int_{1}^{t} s^{3} q_{1}(s) d s<\frac{2(t-1)}{3 \sqrt{3}}, \quad t>1 .
$$

Clearly, (3.41) can be satisfied by infinitely many $q_{1} \in C[1,+\infty)$. Disconjugacy follows by Theorem 2.1.

EXAMPLE 3.12. As a final example using an equation other than the Euler equation for comparison, notice that equation

$$
L y=y^{\prime \prime \prime}+q(t) y=0, q \in C[a, b], q \geqq 0, a<b<+\infty
$$

has property $\mathrm{A}$ on $[a, b]$ provided

$$
\int_{a}^{b}(s-a)^{2} q(s) d s \leqq \frac{1}{2} .
$$

To see this, let $y=y(t)$ be the solution of (3.42) with $y(a)=y^{\prime}(a)=$ $y^{\prime \prime}(a)-1=0$ and suppose there exists $a<t_{0} \leqq b$ with $y^{\prime \prime}\left(t_{0}\right)=0$ and $y^{\prime \prime}>0$ on $\left[a, t_{0}\right)$. Multiplying (3.42) by $y$ and integrating by parts yields

$$
\int_{a}^{t} q(s) y^{2}(s) d s=\frac{\left(y^{\prime}\left(t_{0}\right)\right)^{2}}{2} .
$$

By the Mean Value Theorem, $y(t)<y^{\prime}(t)(t-a), a<t<t_{0}$, so by (3.44) we obtain

$$
\frac{\left(y^{\prime}\left(t_{0}\right)\right)^{2}}{2} \int_{a}^{t_{0}} q(s)(s-a)^{2} d s>\frac{\left(y^{\prime}\left(t_{0}\right)\right)^{2}}{2}
$$

so that $\int_{a}^{t_{0}} q(s)(s-a)^{2} d s>1 / 2$, a contradiction. Now using $q(t) \equiv$ $3 / 2(b-a)^{3}, p(t) \equiv 0$ in Theorem 3.7, it follows by (3.43) that $L y=0$ has property $\mathrm{A}$ (and is disconjugate) on $[a, b]$. Therefore, $L_{1} y=$ $y^{\prime \prime \prime}+q_{1} y=0$ is disconjugate on $[a, b]$ in case either

$$
\begin{aligned}
0 & \leqq \int_{a}^{t}(t-s)(s-a)^{3} q_{1}(s) d s \leqq \frac{3}{2(b-a)^{3}} \int_{a}^{t}(t-s)(s-a)^{3} d s \\
& =\frac{3}{40} \frac{(t-a)^{5}}{(b-a)^{3}}, \quad a<t \leqq b
\end{aligned}
$$

(by Theorem 3.7)

or

$$
\left\{\begin{array}{l}
\int_{a}^{t}(t-s)(s-a)^{3} q_{1}^{+}(s) d s \leqq \frac{3}{40} \frac{(t-a)^{5}}{(b-a)^{3}}, \quad a<t \leqq b \\
\int_{a}^{t}(t-s)(s-a)^{3} q_{1}^{-}(s) d s \leqq \frac{3}{40} \frac{(t-a)^{5}}{(b-a)^{3}}, \quad a<t \leqq b
\end{array}\right.
$$


(by Theorem 2.1).

It is not claimed that the conditions of this example are sharp. The point of this example is that it may occasionally be preferable to test disconjugacy for an equation on a particular interval where a comparison using the Euler equation gives an inferior result. For example, with $p_{1} \equiv 0, q_{1} \equiv k>0$, Theorem 2.2 implies that $y^{\prime \prime \prime}+k y=$ 0 is disconjugate on $[a,+\infty)$ if $k \leqq 2 / 3 \sqrt{3}$ (and hence is disconjugate on any subinterval of $[a,+\infty))$. On the other hand $y^{\prime \prime \prime}+k y=0$ is disconjugate on $[a, b]$ in case $k \leqq k_{0} \equiv 3 / 2(b-a)^{3}$ and $k_{0}>2 / 3 \sqrt{3}$ if $b-a<(9 \sqrt{3} / 4)^{1 / 3}=1.573 \cdots$.

\section{REFERENCES}

1. N. V. Azbelev and Z. B. Caljuk, On the question of the distribution of the zeros of solutions of a third-order linear differential equation, Mat. Sb. [N. S.] 51 (93), 1960, 475-486. Also Amer. Math. Soc. Transl., 42 (1964), 233-245.

2. J. Barrett, Oscillation theory of ordinary linear differential equations, Advances in Math., 3 (1969), 415-509.

3. W. A. Coppel, Disconjugacy, Springer-New York, 1971.

4. L. Erbe, Comparison theorems for second order Riccati equations with applications, SIAM J. Math. Anal., 8 (1977), 1032-1037.

5. - Disconjugacy conditions for the third order linear differential equation, Canad. Math. Bull., 12 (1969), 603-613.

6. G. J. Etgen and C. D. Shih, Disconjugacy of third order differential equations with nonnegative coefficients, J. Math. Anal. Appl., 41 (1973), 420-425.

7. M. Hanan, Oscillation criteria for third order linear differential equations, Pacific J. Math., 11 (1961), 919-944.

8. P. Hartman, Principal solutions of disconjugate $n$-th order linear differential equations, Amer. J. Math., 91 (1969), 306-362.

9. L. K. Jackson, Disconjugacy conditions for linear third order differtntial equations, J. Differential Equations, 4 (1968), 369-372.

10. A. C. Lazer, The behavior of solutions of the differential equation $y^{\prime \prime \prime}+p(x) y^{\prime}+$ $q(x) y=0$, Pacific J. Math., 17 (1966), 435-466.

11. A. Ju. Levin, Some problems bearing on the oscillation of solutions of linear differential equations, Soviet Math. Dokl., 4 (1963), 121-124.

12. C. Swanson, Comparison and Oscillation Theory of Linear Differential Equations, Academic Press, 1968.

Received June 7, 1978 and in revised form January 17, 1979. Research supported by the National Research Council of Canada, Grant No. NRC A-7673.

The University of Alberta

Edmonton, Alberta, Canada T66 2G1 


\section{PACIFIC JOURNAL OF MATHEMATICS}

\section{EDITORS}

DONALD BABBITT (Managing Editor)

University of California

Los Angeles, California 90024

HUgo RossI

University of Utah

Salt Lake City, UT 84112

C. C. MOORE and ANDREW OGG

University of California

Berkeley, CA 94720

\section{J. DUGUNDJI}

Department of Mathematics University of Southern California Los Angeles, California 90007

R. Finn and J. Milgram Stanford University Stanford, California 94305

\section{ASSOCIATE EDITORS}

E. F. BECKENBACH

B. H. NEUMANN

F. WOLF

K. YoshidA

\section{SUPPORTING INSTITUTIONS}

UNIVERSITY OF BRITISH COLUMBIA CALIFORNIA INSTITUTE OF TECHNOLOGY UNIVERSITY OF CALIFORNIA MONTANA STATE UNIVERSITY UNIVERSITY OF NEVADA, RENO NEW MEXICO STATE UNIVERSITY OREGON STATE UNIVERSITY UNIVERSITY OF OREGON
UNIVERSITY OF SOUTHERN CALIFORNIA STANFORD UNIVERSITY UNIVERSITY OF HAWAII UNIVERSITY OF TOKYO UNIVERSITY OF UTAH WASHINGTON STATE UNIVERSITY UNIVERSITY OF WASHINGTON 


\section{Pacific Journal of Mathematics}

Vol. 85, No. $1 \quad$ September, 1979

Ralph Alexander, Metric averaging in Euclidean and Hilbert spaces...... 1

B. Aupetit, Une généralisation du théorème de Gleason-Kahane-Żelazko

pour les algèbres de Banach..............................

Lung O. Chung, Jiang Luh and Anthony N. Richoux, Derivations and

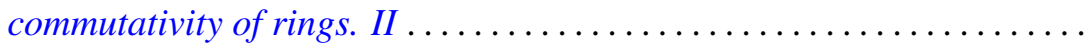

Lynn Harry Erbe, Integral comparison theorems for third order linear

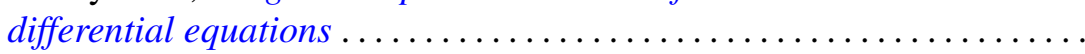

Robert William Gilmer, Jr. and Raymond Heitmann, The group of units of a

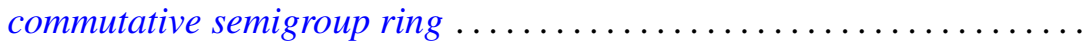

George Grätzer, Craig Robert Platt and George William Sands, Embedding lattices into lattices of ideals ...........................

Raymond D. Holmes and Anthony Charles Thompson, $n$-dimensional area and content in Minkowski spaces ....................... 77

Harvey Bayard Keynes and M. Sears, Modelling expansion in real flows....

Taw Pin Lim, Some classes of rings with involution satisfying the standard

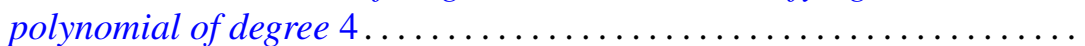

Garr S. Lystad and Albert Robert Stralka, Semilattices having bialgebraic congruence lattices ................................... 131

Theodore Mitchell, Invariant means and analytic actions . . . . . . . . . 145

Daniel M. Oberlin, Translation-invariant operators of weak type ........ 155

Raymond Moos Redheffer and Wolfgang V. Walter, Inequalities involving

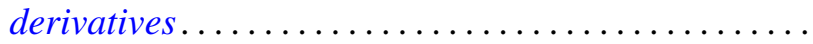

Eric Schechter, Stability conditions for nonlinear products and semigroups ................................

Jan Søreng, Symmetric shift registers ........................ 201

Toshiji Terada, On spaces whose Stone-Čech compactification is $\mathrm{Oz} \ldots \ldots .231$

Richard Vrem, Harmonic analysis on compact hypergroups ... 